\title{
ASPECTOS ATUAIS DA TERAPÊUTICA NEO-ADJUVANTE NO CARCINOMA EPIDERMÓIDE DO ESÔFAGO. REVISÃO DA LITERATURA.
}

\section{New aspects of the neo-adjuvant therapy in esophageal squamous cell carcinoma. A review of medical literature.}

\author{
Valdir TERCIOTI Jr, Luiz Roberto LOPES, João de Souza COELHO NETO, Nelson Adami ANDREOLLO
}

ABCDDV/636

Tercioti V Jr, Lopes LR, Coelho Neto, JS, Andreollo NA. Aspectos atuais da terapêutica neo-adjuvante no carcinoma epidermóide do esôfago. Revisão da Literatura. ABCD Arq Bras Cir Dig 2009;22(1):33-40

RESUMO - Introdução - O câncer de esôfago é o oitavo tipo de câncer mais incidente na população, com alta letalidade a despeito da melhora do tratamento cirúrgico nas últimas décadas. O carcinoma epidermóide tem maior prevalência em vários países em também no Brasil. Sendo assim, estratégias de tratamento neo-adjuvante tornaram-se objeto de estudo em vários centros mundiais de referência. Objetivo - Identificar aspectos atuais da terapêutica neo-adjuvante no tratamento do carcinoma epidermóide do esôfago. Métodos - Revisão bibliográfica de artigos científicos disponíveis no Medline e na base de dados Cochrane cruzando-se os descritores neoplasia esofágicas, cirurgia, quimioterapia, radioterapia. Conclusão - A estratégia da terapêutica neo-adjuvante é cada vez mais utilizada como forma de oferecer melhores resultados tardios na sobrevida e na qualidade de vida dos pacientes portadores de carcinoma do esôfago.

DESCRITORES - Neoplasias esofágicas. Cirurgia. Quimioterapia. Radioterapia.

\section{INTRODUÇÃO}

No mundo, o câncer de esôfago é o oitavo tumor mais frequente, responsável por 462.000 novos casos em 2002 ( $4,2 \%$ do total de casos de câncer) e o sexto tumor mais frequente como causa de morte com 386.000 óbitos (5,7\% do total). A variação geográfica na incidência é muito marcante, com variação de até 20 vezes nas áreas de alto risco da China e de baixo risco na África ocidental. Outras áreas de alto risco relativo são a África oriental, a porção centro-sul da Ásia e o Japão (apenas nos homens) ${ }^{23}$.

As estimativas norte-americanas para o número de casos novos de câncer de esôfago para 2007 foram de 15.560, sendo 12.130 novos casos em homens e $3.430 \mathrm{em}$ mulheres. A mortalidade estimada para 2007 de câncer de esôfago nos EUA foram de 13.940 óbitos no total, sendo 10.900 em homens e 3.040 em mulheres. É certo contudo que ainda lá, apesar dos esforços na melhora do diagnóstico e terapêutica dos diferentes tipos de tumores, houve aumento de $8,1 \%$ dos óbitos por câncer de esôfago em homens entre os anos de 1990 e 2003. Já entre as

Trabalho realizado no Departamento de Cirurgia e Gastrocentro da Faculdade de Ciências Médicas da Universidade Estadual de Campinas - UNICAMP Campinas - SP.

Endereço para correspondência: Nelson Adami Andreollo e-mail: nandreollo@hotmail.com mulheres, no mesmo período, a mortalidade por câncer de esôfago diminuiu em $3,87 \%{ }^{11}$.

Em relação ao câncer de esôfago no Brasil, as estimativas do INCA para 2008 seriam de 7.900 novos casos em homens e 2.650 em mulheres, sendo o oitavo tipo de câncer mais incidente na população brasileira. Além disso, destaca-se a maior incidência deste tipo de neoplasia nas regiões sul e sudeste do Brasil, sendo os Estados de São Paulo e Rio Grande do Sul os com maior incidência, respectivamente, dentro da federação ${ }^{6}$. A taxa de mortalidade por neoplasia de esôfago no Brasil foi de 3,35 por 100.000 habitantes no ano de 2003, segundo dados do Ministério da Saúde ${ }^{20}$.

No Rio Grande do Sul, a incidência de câncer de esôfago está em torno de 14,3/100.000 entre os homens e 4,2/100.00 entre as mulheres, mas estas cifras são mais elevadas em certas regiões, como a micro região Colonial Encosta da Serra Geral, onde se localiza a cidade de Taquara ${ }^{2}$.

Os cânceres de esôfago podem ser classificados, segundo a histologia, em carcinoma epidermóide ou escamoso (CEC) e adenocarcinoma. O primeiro é derivado do epitélio estratificado não-queratinizado, característico da mucosa normal do esôfago. Trata-se do tipo histológico mais comum, ocorre mais frequentemente em homens a 
partir dos 50 anos e acomete principalmente os segmentos médio e inferior (mais de $80 \%$ dos casos) do esôfago. Este tipo de tumor tem íntima correlação entre tabagismo e alcoolismo nos pacientes portadores dessa neoplasia ${ }^{14}$.

Outros fatores do CEC são alimentos, bebidas quentes, vegetais em conservas (compostos nitrosos), agentes infecciosos (vírus papiloma humano), fatores sócio-econômicos (má-nutrição), espru celíaco, síndrome de Plummer-Vinson, deficiências de micronutrientes (riboflavina, vitaminas A, $\mathrm{C}$ e E, zinco e molibdênio), a tilose palmar (uma síndrome autossômica dominante rara), radioterapia prévia, neoplasias de cabeça/pescoço tratadas previamente, acalásia idiopática, megaesôfago chagásico e estenose cáustica prévia $^{25,27}$.

O CEC tem início insidioso, causa disfagia e obstrução esofágica progressiva. Os pacientes se adaptam, em nível subconsciente, à alimentação e à dificuldade de deglutição, alterando progressivamente sua dieta de alimentos sólidos para pastosos e líquidos. Outros sintomas devem ter a atenção dos médicos como leve odinofagia, desconforto retroesternal, sensação de corpo estranho no esôfago proximal, dor epigástrica, anorexia, náuseas, anemia e emagrecimento sem causa aparente.

A esofagoscopia, complementada pela biópsia e citologia, constitui o mais eficiente método para diagnóstico do câncer de esôfago, sendo o primeiro exame a ser indicado diante de um paciente com disfagia.

Assim, esta revisão tem finalidade de identificar aspectos atuais da terapêutica neo-adjuvante no tratamento do carcinoma epidermóide do esôfago.

\section{MÉTODOS}

A terapêutica do carcinoma de esôfago deve ser dividida inicialmente em: a) a paliativa em que se incluem a quimioterapia (Qtx) e/ou radioterapia (Rtx) exclusivas e o uso de próteses endoscópicas; e b) radical com intenção curativa, onde se incluem a ressecção cirúrgica, a Qtx e/ou Rtx neo-adjuvante e a Qtx e/ou Rtx adjuvantes.

\section{Terapêutica neo-adjuvante}

Apesar do progresso nos últimos anos no tratamento cirúrgico do carcinoma do esôfago, a sobrevida a longo prazo continuava desapontadora, mesmo após ressecção completa. Apenas uma minoria obtém ressecção macro e microscópica completa do tumor primário, incluindo seus sítios de drenagem linfática (ressecção R0, como definido pela UICC). No momento do diagnóstico, cerca de dois terços dos pacientes já apresentam tumores localmente avançados que cresceram além da parede esofágica, ou seja, tumores invadindo a adventícia (T3) e estruturas adjacentes $(\mathrm{T} 4)^{30}$.

A radioterapia no câncer de esôfago é utilizada há muitos anos, com bons resultados, objetivando o tratamento definitivo (radioterapia exclusiva) como também o tratamento neo-adjuvante a fim de melhorar o controle loco-regional da doença. Além disso, a radioterapia exclusiva tem como objetivo preservar os órgãos de ressecção, tais como a laringe no câncer do esôfago cervical ou o estômago no câncer do esôfago dista ${ }^{24}$. Consequentemente, estratégias terapêuticas multidisciplinares empregando princípios adjuvante (tratamento pós-operatório após ressecção completa) e/ ou neo-adjuvante (pré-operatório) teem recebido atenção crescente pela comunidade científica.

A terapia neo-adjuvante deve ser realizada três a quatro semanas antes da operação e apresenta como vantagens a possível melhora na ressecção completa do tumor e o aumento na sobrevida global e livre de doença. A seu favor, existem algumas vantagens clínicas e teóricas, a saber: a) vasos sanguíneos e linfáticos íntegros, que possibilitam concentração de droga efetiva nas áreas peri-tumorais com oxigenação tumoral e subsequente radiosensibilidade preservadas; b) melhor estado clínico ("performance status") do paciente quando comparado com a sua situação pós-operatória por meio de melhora na nutrição (aumento da ingestão no pré-operatório), o que possibilita melhor administração de Qtx combinada baseada em cisplatina mais agressiva; c) melhora do estado clínico dos pacientes respondedores à terapia neo-adjuvante no período pós-operatório; d) redução tumoral e, portanto, aumento da probabilidade de ressecção completa e a radioterapia pode também atingir linfonodos mediastinais acometidos pela doença; e) a terapia pré-operatória poderia desvitalizar células tumorais e minimizar os riscos de contaminação intra-operatória e implantes de células tumorais.

Os objetivos primários da modalidade combinada de tratamento (Qtx e Rtx) no período pré-operatório são o aumento do controle local - através da intensificação da Rtx com Qtx concomitante (radio-sensibilizadora) -, e a erradicação de micrometástases clinicamente ocultas através da Qtx sistêmica ${ }^{13}$.

As desvantagens da terapêutica neo-adjuvante seriam: a) retardar o procedimento cirúrgico; b) causar edema e fibrose no mediastino podendo dificultar a operação; c) propiciar o acúmulo de secreções pulmonares no préoperatório e deteriorar a ventilação pulmonar; d) poder causar anemia e leucopenia. O aparecimento de fístula esôfago-brônquica é ocorrência pouco frequente.

\section{DISCUSSÃO}

Várias publicações nos últimos anos abordaram estratégias neste sentido com resultados nem sempre concordantes.

Le Prise et al. ${ }^{15}$ em 1994, em estudo randomizado na França avaliaram o benefício da quimioradioterapia neoadjuvante (dois ciclos de 5-fluorouracil e cisplatina com 20 Gy de radioterapia concomitantes) comparados a um grupo controle (operação exclusiva). Os critérios de inclusão dos pacientes foram: CEC do esôfago, idade menor que 70 anos, estado clínico abaixo de 2 da classificação da Organização Mundial de Saúde, tempo de sobrevida estimada maior que três meses e nenhum tratamento prévio para câncer. Os critérios de exclusão foram: perda de peso maior que $15 \%$, a presença de fístula tráqueo-esofágica, metástases viscerais e infecção descontrolada. Foram incluídos 86 pacientes 
(41 para o grupo de quimioradioterapia, 45 para o grupo controle). Os grupos foram comparáveis para idade, sexo, localização tumoral, tamanho tumoral e estadiamento. A mortalidade operatória foi de $8,5 \%$ e $7 \%$, respectivamente, para cada grupo com período de internação hospitalar de 27 dias para ambos os grupos. A sobrevida a longo prazo não foi significativamente diferente entre os grupos, com $47 \%$ dos pacientes vivos após um ano em ambos os grupos. A conclusão dos autores foi de que o tratamento neoadjuvante não alterou a mortalidade operatória ou o tempo de sobrevida para os pacientes com CEC do esôfago.

Ancona et al. ${ }^{1}$ em 1997, em estudo prospectivo não randomizado na Itália avaliaram a quimioterapia como primeira linha de tratamento no CEC do esôfago torácico localmente avançado (T4, qualquer N, M0) a fim de melhorar a taxa de ressecção e a sobrevida a longo prazo. Entre janeiro de 1983 a dezembro de 1991, 163 pacientes consecutivos com CEC do esôfago torácico, presumivelmente T4 (grupo A), receberam média de 2,5 ciclos (intervalo, 1 a 6 ciclos) de quimioterapia com cisplatina $(100 \mathrm{mg} / \mathrm{m} 2$ no dia 1$)$ e 5 -fluorouracil $(1000 \mathrm{mg} / \mathrm{m} 2 / \mathrm{dia}$, em infusão contínua nos dias 1 a 5). A quimioterapia foi seguida por procedimento cirúrgico quando adequada diminuição tumoral foi obtida. A toxicidade observada da quimioterapia foi de graus 0 a 2 da Organizaçao Mundial da Saúde em $80 \%$ dos casos, mas houve três óbitos (1,9\%). O re-estadiamento sugeriu diminuição tumoral em 101 de 163 pacientes $(62 \%)$, mas apenas $85(52 \%)$ foram submetidos à ressecção cirúrgica sendo completa ou R0 em 52 (32\%) e incompleta ou R1-2 em 33. A mortalidade pós-operatória global foi de $11,7 \%$ (10 de 85 pacientes), a morbidade de $41 \%$ (35 de 85 pacientes). A resposta patológica completa foi documentada em seis pacientes e diminuição tumoral significativa para estádios patológicos I, IIA ou IIB ocorreram em 25 pacientes adicionais. A sobrevida global em cinco anos foi de $11 \%$ (média, 11 meses). Após a ressecção cirúrgica, a sobrevida em cinco anos foi de $20 \%$ (média, 16 meses); nenhum dos pacientes não-respondedores sobreviveu quatro anos após o tratamento paliativo sem ressecção (sobrevida média, cinco meses). A taxa de sobrevida em cinco anos dos 52 pacientes submetidos à ressecção R0 foi de 29\% (média, 23 meses). Estratificando os pacientes de acordo com as classificações R, pT, pN e estadio patológico, as curvas de sobrevida foram comparáveis aos dados correspondentes obtidos de um grupo B de 587 pacientes com câncer de esôfago potencialmente ressecáveis que foram submetidos a operação isolada durante o mesmo período do estudo. Além disso, os resultados foram melhores em comparação com 136 pacientes prévios ou subsequentes com tumor localmente avançado que não receberam quimioterapia neo-adjuvante (grupo C). Nestes pacientes, a taxa de ressecção R0 foi de $7 \%$ e a sobrevida em cinco anos de 3\% (média, cinco meses). Os autores deste estudo concluíram que embora não sendo trabalho randomizado, os resultados sugerem que no CEC localmente avançado de esôfago, a quimioterapia de primeira linha aumenta a taxa de ressecção e melhora a sobrevida a longo prazo. Nos que respondem ao tratamento e são submetidos à ressecção cirúrgica R0, o prognóstico depende do estadiamento patológico final e não no clínico pré-tratamento.

Jones et al. ${ }^{12}$ em 1997 nos EUA avaliaram a quimioradioterapia neo-adjuvante seguido pela esofagectomia. No período de 1988 a 1996, 166 pacientes consecutivos com câncer de esôfago foram avaliados; 66 participaram de um protocolo de quimioterapia (5-fluorouracil, cisplatina) concomitante com radioterapia (45 Gy) seguidos por esofagectomia, sendo que 54 pacientes completaram o protocolo de tratamento. A toxicidade associada com a quimioradioterapia neo-adjuvante foi mínima. A sobrevida atuarial em 12, 24 e 36 meses foi de $59 \%$, $42 \%$ e $32 \%$, respectivamente. A taxa de resposta patológica completa foi de $41 \%$, com sobrevidas em 12, 24 e 36 meses de $77 \%$, $50 \%$ e $45 \%$, respectivamente, enquanto os pacientes que não apresentaram resposta patológica completa tiveram sobrevidas de $46 \%, 35 \%$ e $23 \%$. A diferença na sobrevida entre o grupo com e sem resposta patológica completa não foi estatisticamente significativa $(P=0,13)$, mas a na sobrevida livre de doença foi estatisticamente significativa $(P=0,007)$. Os autores concluíram que o tratamento trimodal no câncer de esôfago pode obter sobrevida a longo prazo em alguns pacientes dependendo da resposta tumoral a quimioradioterapia.

Bosset et al. ${ }^{3}$ em 2007, em estudo multicêntrico randomizado na Europa, compararam a quimioradioterapia neo-adjuvante seguida por operação (terapia combinada) com o tratamento cirúrgico isolado nos pacientes com estágios I e II do CEC de esôfago. A quimioradioterapia neo-adjuvante consistiu em dois ciclos, cada um com uma dose de 18,5 Gy administrado em cinco frações de 3,7 Gy, e $80 \mathrm{mg} / \mathrm{m} 2$ de cisplatina administrada de 0 a 2 dias antes do primeiro dia da radioterapia. O plano cirúrgico consistiu em esofagectomia e gastrectomia proximal por meio de incisões abdominal e torácica direita a serem realizadas imediatamente após a randomização no grupo de operação isolada e após duas a quatro semanas após o término da quimioradioterapia neo-adjuvante no grupo de terapia combinada. Um total de 297 pacientes participou do estudo; 11 foram excluídos e quatro perdidos no seguimento. Dos 282 pacientes remanescentes, 139 ficaram no grupo de operação isolada e 143 no grupo de terapia combinada. Após seguimento médio de 55,2 meses, não foi observada diferença estatística significativa na sobrevida global; a sobrevida média para ambos os grupos foi de 18,6 meses. Em comparação ao grupo tratado com operação isolada, o grupo de terapia combinada apresentou maior sobrevida livre de doença $(P=0,003)$, intervalo maior livre de doença local $(P=0,01)$, taxa menor de mortes relacionadas ao câncer $(P=0,002)$ e frequência maior de ressecções curativas $(P=0,017)$. Entretanto, houve mais mortes pósoperatórias $(P=0,012)$ no grupo de terapia combinada. Foram identificados três fatores prognósticos na influência da sobrevida na análise multivariada: o estágio da doença baseado na TC; a localização do tumor; e se a ressecção cirúrgica foi curativa. A conclusão deste trabalho foi de que nos pacientes com CEC do esôfago, a quimioradioterapia neo-adjuvante não melhora a sobrevida global, mas pro- 
longa a sobrevida livre de doença e a livre de doença local.

Coia et al. ${ }^{5}$ em 2000 nos EUA examinaram os registros de pacientes com câncer de esôfago tratados com radioterapia entre 1992 a 1994 a fim de determinar as práticas de cuidados e resultados naquele país e compará-los com outros trabalhos clínicos. Uma pesquisa nacional em 63 instituições foi realizada e informação específica foi coletada de 400 pacientes com CEC (62\%) ou adenocarcinoma (37\%) do esôfago torácico que receberam radioterapia como parte do tratamento primário ou adjuvante. Os pacientes foram estadiados de acordo com o sistema da "American Joint Committe on Cancer" modificado de 1983, sendo 15\% dos pacientes com estádio I, $40 \%$ com estádio II e $30 \%$ com estádio III. Do total de pacientes, $26 \%$ foram submetidos à esofagectomia, $75 \%$ receberam quimioterapia e $84 \%$ receberam radioterapia e quimioterapia concomitantes. Como resultado, a quimioradioterapia neo-adjuvante obteve sobrevida em dois anos maior comparado com a quimioradioterapia definitiva sem significância estatística (63\% versus $39 \% ; P=0,11$ ), confirmando o valor da quimioradioterapia no tratamento do câncer de esôfago.

Toita et al. ${ }^{26}$ em 2001 no Japão avaliaram a aplicabilidade e os resultados com a quimioradioterapia concomitantes no tratamento do CEC do esôfago torácico, sem a utilização de operação. A radioterapia consistiu em irradiação regional (estendendo-se da fossa supraclavicular para a área paracardíaca) com 39,6 Gy seguidos por alta dose de radiação externa complementar até 66,6 Gy $(1,8$ $\mathrm{Gy} / \mathrm{dia}$, cinco vezes por semana). A quimioterapia consistiu em cisplatina em infusão em duas horas $(80 \mathrm{mg} / \mathrm{m} 2$ no dia 1) e infusão contínua de 5 -fluorouracil $(800 \mathrm{mg} / \mathrm{m} 2 /$ dia nos dias 2-6) administrados concomitantemente com a radioterapia, durante três a quatro semanas, em dois ciclos. Participaram deste estudo 30 pacientes (três no estádio I, 11 no II, 16 no III), sendo que 21 pacientes (70\%) completaram o tratamento planejado. Nos pacientes idosos ( $\geq 70$ anos), quatro de seis desistiram. Foram observadas toxicidades graus 3 e 4 ("National Cancer Institute Common Toxicity Criteria" versão 2.0) em 20 (67\%) e 3 (10\%) pacientes, respectivamente. As maiores toxicidades foram hematológicas, gastrointestinais (por exemplo, náuseas e esofagites) e pulmonares. Não houve toxicidade grau 5 (fatal). O tempo de seguimento médio dos pacientes sobreviventes foi de 27 meses (intervalo: 9-49 meses). O tempo de sobrevida média foi de 21 meses. As taxas de sobrevida em um e dois anos foram de $65 \%$ e $49 \%$ para todos os 30 pacientes. A incidência de estenose esofágica foi de $21 \%$ (graus 1 e 2 do "Radiation Therapy Oncology Group"). Nenhum paciente apresentou fistula. A conclusão do estudo foi de que apesar da baixa aderência ao tratamento dos pacientes idosos e das toxicidades frequentes, a quimioradioterapia concomitante utilizada apresentou resultado favorável no CEC do esôfago torácico.

Hennequin et al. ${ }^{10}$ também em 2001 na França avaliaram os resultados da quimioradioterapia concomitantes com ou sem a operação nos pacientes com CEC localmente avançados do esôfago (T3 e/ou envolvimento linfonodal). Foram avaliados 112 pacientes submetidos à quimiora- dioterapia seguido por uma reavaliação; a operação foi realizada ou, então, a quimioradioterapia foi continuada, baseando-se na regressão tumoral e no estado clínico geral do paciente. A quimioradioterapia consistiu em administração concomitante de 5-fluorouracil $(1 \mathrm{~g} / \mathrm{m} 2$ nos dias 1 a 3), cisplatina ( $50 \mathrm{mg} / \mathrm{m} 2$ nos dias 1 e 2) e irradiação externa até uma dose de 40 ou 43,2 Gy. Após período de repouso de quatro semanas, a esofagectomia radical (dissecção linfonodal em dois campos) ou um novo ciclo de quimioradioterapia (até dose total de $65 \mathrm{~Gy}$ ) foi realizada. Resposta clínica completa foi obtida em $25,7 \%$ dos pacientes e resposta parcial em 45,9\%. Cinquenta pacientes foram operados, mas apenas 38 tiveram esofagectomia. A mortalidade pós-esofagectomia foi de 5,3\%. Foi obtida resposta patológica completa em $23,7 \%$. As taxas de sobrevida em dois e cinco anos foram, respectivamente, $41,5 \%$ e 28,6\% para o grupo como um todo. Após a análise multivariada, os fatores prognósticos para sobrevida foram o índice de Karnofsky, a esofagectomia e a resposta à quimioradioterapia. A sobrevida para os pacientes que experimentaram resposta parcial à quimioradioterapia foi de $49,1 \%$ para aqueles operados e $23,5 \%$ para os tratados cirurgicamente $(P=0,003)$. Não houve benefício evidente para o pequeno número de pacientes assim tratados após a resposta completa à quimioradioterapia. A toxicidade, essencialmente hematológica, foi moderada. A conclusão do trabalho foi de que para os CEC localmente avançados, a esofagectomia, após quimioradioterapia concomitantes, pode melhorar a taxa de sobrevida, especialmente para os pacientes que responderam parcialmente a esta última.

Neste mesmo ano de 2001, Urba et al. ${ }^{28}$ em trabalho na Universidade de Michigan nos EUA realizaram um estudo randomizado com 100 pacientes com carcinoma do esôfago para receber operação isolada (grupo I) ou quimioradioterapia neodjuvante (grupo II) com cisplatina $20 \mathrm{mg} / \mathrm{m} 2 / \mathrm{d}$ no dias 1 a 5 e 17 a 21 , fluorouracil $300 \mathrm{mg} / \mathrm{m} 2 / \mathrm{d}$ no dias 1 até 21 e vinblastina $1 \mathrm{mg} / \mathrm{m} 2 / \mathrm{d}$ nos dias 1 a 4 e 17 a 20 . A radioterapia consistiu em 1,5 Gy/sessão diariamente durante 21 dias, até dose total de $45 \mathrm{~Gy}$. A esofagectomia transhiatal foi realizada com anastomose esôfago-gástrica cervical aproximadamente no dia 42 . Houve seguimento médio de 8,2 anos, sem diferença significativa na sobrevida entre os grupos de tratamento. A sobrevida média foi de 17,6 meses no grupo I e 16,9 meses no grupo II. A sobrevida em três anos foi de $16 \%$ no grupo I e $30 \%$ no grupo II $(P=0,15)$. Este estudo foi desenhado para detectar diferença relativamente grande no aumento da sobrevida média de um ano até 2,2 anos, com ao menos poder estatístico de $80 \%$. A conclusão dos autores foi de que a quimioradioterapia neo-adjuvante versus operação isolada não demonstrou diferença significativa na sobrevida.

Nakadi et al. ${ }^{22}$ ainda em 2001 em estudo prospectivo na Bélgica avaliaram o tratamento multimodal (quimioradioterapia + cirurgia) no CEC avançado de esôfago (estadio III) comparado a um grupo controle submetido a tratamento cirúrgico isolado. Sessenta e um pacientes com CEC avançado de esôfago foram submetidos à quimioterapia neo-adjuvante (5-fluorouracil e cisplastina) com 45 
Gy de radioterapia concomitante durante cinco semanas, seguido por esofagectomia transtorácica quatro a cinco semanas após o final do tratamento neo-adjuvante. Trinta e oito pacientes foram submetidos à operação, sendo que 37 tiveram esofagectomia (ressecabilidade: $97 \%$ no grupo multimodal e $84 \%$ no grupo controle; $P=0,07$ ). A taxa de ressecção completa R0 foi de $78 \%$ no grupo multimodal e de $56 \%$ no grupo controle $(P<0,03)$. Onze pacientes não tiveram tumor residual na peça cirúrgica (resposta patológica completa de $30 \%$ ). A taxa de mortalidade operatória foi de $19 \%$ no grupo multimodal e $8,8 \%$ no grupo controle. A sobrevida média global nos pacientes operados foi de 21 meses, com taxa de sobrevida em cinco anos de $11 \%$ (14 \% no grupo controle; não significativo). As taxas de sobrevida em três e cinco anos foram de $34 \%$ nos pacientes que apresentaram resposta patológica completa e de $5 \%$ e 0 , respectivamente, nos pacientes com resposta patológica incompleta $(P<0,05)$. A sobrevida média foi de 28 meses nos pacientes com resposta patológica completa e 19 meses no grupo com resposta patológica incompleta. $\mathrm{O}$ estudo concluiu que a quimioradioterapia neo-adjuvante no CEC avançado de esôfago parece causar aumento na ressecabilidade cirúrgica e da taxa de ressecção R0, aumento da taxa de resposta patológica completa, aumento da sobrevida apenas no grupo com resposta patológica completa, às expensas de aumento inadequado na mortalidade operatória.

Urschel et al. ${ }^{29}$ em 2002 realizaram metanálise no Canadá e avaliaram nove estudos controlados e randomizados que envolveram 1.116 pacientes. Os resultados foram expressos em razão de chances ("odds ratio") entre quimioradioterapia neo-adjuvante e operação versus operação isolada (tratamento versus controle; valores $<1$ favorecem a quimioradioterapia neo-adjuvante) seguidos pelos intervalos de confiança com nível de significância de $95 \%$. Os "odds ratio" foram $0,79(0,59-1,06 ; P=0,12)$ para a sobrevida em um ano; $0,77(0,56-1,05 ; P=0,10)$ para a em dois anos; $0,66(0,47-0,92 ; P=0,016)$ para a em três anos; $2,50(1,05-5,96 ; P=0,038)$ para a taxa de ressecção; 0,53 $(0,33-0,85 ; P=0,007)$ para a taxa de ressecção completa; $1,72(0,96-3,07 ; P=0,07)$ para a mortalidade operatória; $1,63(0,99-2,68 ; P=0,053)$ para a mortalidade de todo o tratamento; $0,38(0,23-0,63 ; P=0,0002)$ para a recorrência tumoral loco-regional; $0,88(0,55-1,41 ; P=0,60)$ para a recorrência tumoral à distância; e $0,47(0,16-1,45 ; P=0,19)$ para a recorrência tumoral total. Resposta patológica completa à quimioradioterapia ocorreu em $21 \%$ dos pacientes. O benefício da sobrevida em três anos foi mais pronunciada quando a quimioterapia e radioterapia foram aplicadas concomitantemente ("odds ratio" 0,$45 ; 0,26-0,79 ; P=0,005$ ) em comparação com a aplicação sequencial ("odds ratio" $0,82 ; 0,54-1,25 ; P=0,36)$. Os autores da metanálise concluíram que comparado com o tratamento cirúrgico isolado, a quimioradioterapia neo-adjuvante e procedimento cirúrgico associado melhoraram a sobrevida em três anos e diminuíram a recorrência tumoral loco-regional. Além disso, foi associada com taxa menor de ressecção esofágica, mas com taxa de ressecção completa maior (R0). Houve tendência estatisticamente não significativa de aumento na mortalidade do tratamento com o uso da quimioradioterapia neo-adjuvante. E, finalmente, a administração concomitante da quimioterapia e radioterapia neo-adjuvantes foi superior à administração sequencial.

Makary et al. ${ }^{17}$ em 2003 nos EUA avaliaram retrospectivamente 116 pacientes submetidos à esofagogastrectomia com reconstrução devido a carcinoma do esôfago ou da junção gastroesofágica durante um período de 10 anos (entre janeiro de 1990 a junho de 2001). Destes pacientes, 40 foram submetidos à quimioradioterapia neo-adjuvante seguido por operação. A mortalidade hospitalar neste grupo foi de $7,5 \%$, a resposta patológica completa de $37,5 \%$ e as taxas de sobrevida global em três e cinco anos de $47 \%$ e $38 \%$, respectivamente. A sobrevida em cinco anos dos 15 pacientes com resposta patológica completa foi de $85 \%$. Houve cinco pacientes que foram submetidos à terapêutica neo-adjuvante com uma única modalidade (quatro com quimioterapia e um com radioterapia) seguidos por operação, e nenhum daqueles pacientes sobreviveu até três anos. Do total de pacientes, 71 foram submetidos à operação sem tratamento neo-adjuvante. A mortalidade hospitalar neste grupo foi de $1,4 \%$, com sobrevidas em três e cinco anos de $21 \%$ e $17 \%$, respectivamente, com sobrevida a longo prazo diminuída em comparação com o grupo da terapêutica neo-adjuvante, apesar da observação de que os pacientes submetidos à quimioterapia neo-adjuvante apresentavam tamanho tumoral maior no início do tratamento $(5,5 \pm 0,4$ $\mathrm{cm}$ versus $3,8 \pm 0,2 \mathrm{~cm} ; P=0,002)$. Os CEC pareceram ser mais responsivos à quimioradioterapia neo-adjuvante seguido pela operação do que os adenocarcinomas, com taxa de resposta patológica completa de $44,4 \%$ versus $35,5 \%$; entretanto, a sobrevida em cinco anos destes pacientes "respondedores completos" não foram diferentes estatisticamente ( $100 \%$ e $78 \%$, respectivamente; $P=0,97)$. Os autores concluíram que a esofagogastrectomia em conjunto com a terapêutica neo-adjuvante resulta em melhora na sobrevida quando comparado com a operação isolada $(P<0,01)$, embora possa existir aumento na mortalidade peri-operatória associada com a terapêutica neo-adjuvante.

Lee et al. ${ }^{16}$ também em 2003 desenvolveram estudo prospectivo de fase II na Coréia do Sul e avaliaram a quimioradioterapia neoadjuvante em CEC de esôfago ressecável no período de maio de 1993 a março 1996. Um total de 88 pacientes foram incluídos e tratados com dois ciclos de quimioterapia (cisplatina $60 \mathrm{mg} / \mathrm{m} 2$ no dia 1 e 5 -fluorouracil $1000 \mathrm{mg} / \mathrm{m} 2 /$ dia nos dias 2-6) com radioterapia hiperfracionada (48 Gy em 40 sessões durante quatro semanas) seguidos por esofagectomia ou quimioradioterapia definitiva compreendendo quatro ciclos de cisplastina/5-fluorouracil e 12 Gy adicional de teleterapia e 9 Gy de braquiterapia. A resposta clínica e a diminuição do estadiamento foram obtidos em $83 \%$ e $42 \%$ dos pacientes, respectivamente. Com seguimento médio de 77 meses, a sobrevida média foi de 18 meses com taxa de sobrevida em cinco anos de $23 \%$. As respostas clínicas à quimioradioterapia e operação foram fatores prognósticos independentes na sobrevida global. Entre os pacientes do grupo cirúrgico $(n=52), 41(79 \%)$ pacientes efetivamente foram operados 
e 36 tiveram ressecção com taxa de resposta patológica completa de $43 \%$. Quando comparados a um grupo de controle histórico $(\mathrm{n}=40)$, houve significativo benefício na sobrevida com o tratamento multimodal $(P=0,04)$. $\mathrm{O}$ tratamento multimodal foi possível e sua eficácia foi promissora, especialmente quando a ressecção cirúrgica foi realizada. Os autores concluíram que o benefício terapêutico da quimioradioterapia deverá ser averiguada em grandes estudos randomizados bem desenhados no futuro.

Fiorica et al. ${ }^{6}$ em 2004 realizaram uma metanálise na Itália, incluíram seis estudos controlados randomizados e concluíram que nos pacientes com câncer de esôfago ressecável, a quimioradioterapia neo-adjuvante com operação reduz significativamente a mortalidade no terceiro ano comparado ao tratamento cirúrgico isolado. Entretanto, a mortalidade pós-operatória foi significativamente aumentada pela quimioradioterapia neo-adjuvante.

Zhang et al. ${ }^{31}$ em 2005 na Austrália avaliaram 115 pacientes submetidos à esofagectomia entre janeiro de 1999 a janeiro de 2004, sendo 49 pacientes com adenocarcinoma e 26 com CEC. Destes, 56 foram submetidos à quimioradioterapia neo-adjuvante (dois ciclos de cisplatina e 5-fluorouracil com uma dose de 45 Gy de radioterapia) seguido por esofagectomia. Os demais 59 foram à esofagectomia isoladamente. Os resultados foram observados prospectivamente e o seguimento disponível para todos os pacientes. A quimioradioterapia neoadjuvante obteve diminuição tumoral em 43\%, 43\% e $46 \%$ dos pacientes, de acordo com as classificações T, N e TNM, respectivamente. A quimioradioterapia resultou em resposta patológica completa em sete pacientes (13\%). A morbidade cirúrgica foi de $37 \%$ (42/115), e a mortalidade intra-hospitalar de 5\% (6/115). Não houve diferenças entre os pacientes que fizeram e não fizeram a quimioradioterapia neo-adjuvante em relação ao grau de ressecção, à mortalidade peri-operatória e a morbidade pós-operatória. A sobrevida em quatro anos foi de $33 \%$ no grupo da quimioradioterapia neo-adjuvante, comparado com $19 \%$ para os pacientes submetidos à operação isoladamente. A administração de quimioradioterapia neo-adjuvante nos pacientes com CEC obteve cerca de $50 \%$ de diminuição tumoral, e resposta patológica completa ocorreu em alguns destes pacientes. Não houve aumento da morbidade pós-operatória e da mortalidade peri-operatória com o uso da quimioradioterapia neoadjuvante. Neste estudo não-randomizado, houve tendência para sobrevida melhor no grupo da quimioradioterapia neo-adjuvante.

Nabeya et al. ${ }^{21}$ também em 2005, em estudo prospectivo não randomizado no Japão, avaliaram o efeito da quimioradioterapia neo-adjuvante (5-fluorouracil + cisplatina + 46 Gy radioterapia concomitantes) na esofagectomia com linfadenectomia em três campos. Entre 1998 e 2002, 83 pacientes com CEC de esôfago inicialmente ressecável foram prospectivamente alocados em dois grupos, de acordo com o estádio clínico da metástase linfonodal. Dezenove clinicamente com múltiplas metástases linfonodais foram submetidos à quimioradioterapia neo-adjuvante seguidos por esofagectomia curativa com linfadenectomia em três campos (grupo quimioradioterapia). Os demais 64 clinica- mente sem metástases linfonodais foram submetidos diretamente a esofagectomia curativa com linfadenectomia em três campos (grupo controle). Embora a taxa de mortalidade global tenha sido significativamente maior no grupo quimioradioterapia, não houve mortalidade intra-hospitalar em ambos os grupos. Os pacientes sem metástases patológicas linfonodais múltiplas do grupo quimioradioterapia mostraram sobrevida livre de doença significativamente melhor do que os com metástases patológicas linfonodais múltiplas do grupo controle ou do quimioradioterapia. Portanto, o significativo benefício na sobrevida da quimioradioterapia em adição a esofagectomia com linfadenectomia em três campos não foi confirmado, embora tenha sido vantajoso, sem aumento da mortalidade, para alguns pacientes que responderam bem à quimioradioterapia. $\mathrm{O}$ trabalho conclui que a quimioradioterapia pode ser escolha inicial de tratamento para CEC de esôfago ressecável clinicamente com múltiplas metástases linfonodais, e a previsão de resposta à quimioradioterapia como também o desenvolvimento de tratamentos alternativos para a recorrência hematogênica podem obter melhora na sobrevida nesta modalidade de tratamento trimodal.

Burmeister et al. ${ }^{4}$ ainda em 2005 nos EUA, em estudo controlado randomizado de fase III, incluíram 128 pacientes aleatoriamente para tratamento cirúrgico isolado e 128 pacientes também aleatoriamente para operação após quimioradioterapia neo-adjuvante $(80 \mathrm{mg} / \mathrm{m} 2$ de cisplatina no dia 1 , $800 \mathrm{mg} / \mathrm{m} 2$ de fluorouracil nos dias 1 a 4, com radioterapia concomitante de 35 Gy aplicados em 15 sessões). Não foram encontradas diferenças significativas da sobrevida livre de doença e da sobrevida global (taxa de risco 0,82 $[0,61$ a 1,10$]$ e 0,89 [0,67 a 1,19], respectivamente). O grupo da quimioradioterapia neo-adjuvante apresentou maior número de ressecções com margens livres que o grupo da operação isolada (103 de 128 [80 \%] versus 76 de 128 [59 $\%$ ], $P=0,0002)$ e apresentou menor número de linfonodos positivos (44 de 103 [43\%] versus 69 de 103 [67\%], $P=0,003)$. A análise de subgrupos mostrou que pacientes com CEC apresentaram sobrevida livre de doença maior com a quimioradioterapia neo-adjuvante em comparação com os não-CEC (taxa de risco $0,47[0,25-0,86]$ versus $1,02[0,72-1,44])$. Entretanto, os autores ressalvaram que o estudo não possuiu poder estatístico para avaliar a real magnitude deste benefício neste subgrupo.

Greer et al. ${ }^{9}$ também em 2005 nos EUA, concluíram uma metanálise após a avaliação de seis estudos randomizados (quimioradioterapia neo-adjuvante seguido por operação versus operação isolada) constatando que a quimioradioterapia neoadjuvante está associada com pequena, estatisticamente não significativa, melhora na sobrevida global. Se este benefício é suficiente para sobrepujar os custos e riscos associados com à quimioradioterapia neoadjuvante, somente estudos randomizados maiores poderão esclarecer no futuro.

Malthaner et al. ${ }^{19}$ em 2004 por meio de metanálise no Canadá avaliaram 34 trabalhos randomizados e seis metanálises envolvendo 13 diferentes combinações de tratamento neo-adjuvante e/ou adjuvante na neoplasia de 
esôfago ressecável e concluíram que o tratamento cirúrgico isolado (ou seja, sem terapêutica neo-adjuvante ou adjuvante) deve ser recomendado como o padrão de tratamento no câncer de esôfago torácico ressecável.

Malthaner et al. ${ }^{18}$ novamente em 2006 finalizaram outra metanálise do Banco de Dados Cochrane de Revisões Sistemáticas ("Cochrane Database of Systematic Reviews") sobre o papel da quimioterapia neo-adjuvante no câncer de esôfago torácico ressecável e concluíram que a quimioterapia neo-adjuvante poderia oferecer vantagem na sobrevida comparado ao tratamento cirúrgico isolado para o câncer de esôfago torácico ressecável, mas as evidências foram inconclusivas. Além disso, acrescentaram que havia evidências de toxicidade e mortalidade pré-operatória associadas com a quimioterapia.

Gebski et al. ${ }^{8}$ em 2007 em metanálise na Austrália com o objetivo de esclarecer os benefícios da quimioradioterapia ou quimioterapia neoadjuvantes em comparação com a operação isolada identificaram 10 trabalhos randomizados de quimioradioterapia neo-adjuvante versus operação isolada $(n=1209)$ e oito trabalhos de quimioterapia neo-adjuvante versus operação isolada $(\mathrm{n}=1724)$ em pacientes com câncer de esôfago operável. O risco relativo para todas as causas de morte entre os grupos quimioradioterapia neoadjuvante versus operação isolada foi de 0,81 (intervalo de confiança:0,70-0,93; $P=0,002$ ), correspondendo a diferença absoluta de $13 \%$ na sobrevida aos dois anos, com resultados similares para os diferentes tipos histológicos de tumores: $0,84(0,71-0,99 ; P=0,04)$ para CEC e 0,75 $(0,59-0,95 ; P=0,02)$ para adenocarcinoma. $\mathrm{O}$ risco relativo para quimioterapia neo-adjuvante foi de $0,90(0,81-1,00$; $P=0,05)$, o que indica diferença absoluta de $7 \%$ na sobrevida aos dois anos. Não houve efeito significativo nas causas de mortalidade nos pacientes com CEC submetidos à quimioterapia - risco relativo $0,88(0,75-1,03 ; P=0,12)$, embora houve benefício significativo para aqueles com adenocarcinoma - risco relativo $0,78(0,64-0,95 ; P=0,014)$. A conclusão da metanálise foi de que benefício foi evidente para a quimioradioterapia neo-adjuvante e, em menor grau, para a quimioterapia neo-adjuvante nos pacientes com adenocarcinoma de esôfago.

Ainda em relação a esta metanálise, a discussão dos potenciais benefícios da quimioradioterapia neo-adjuvante ou da quimioterapia adjuvante para cada paciente com câncer de esôfago operável é compreensível. Foram observados benefícios similares tanto para quimioradioterapia neoadjuvante como para quimioterapia neo-adjuvante nos pacientes com adenocarcinoma, enquanto foram observados benefícios somente com quimioradioterapia neo-adjuvante nos pacientes com CEC. A quimioradioterapia poderia obter redução tumoral da lesão primária como também ocasionar efeitos nos linfonodos mediastinais. Os pacientes com CEC apresentam drenagem linfonodal muito mais imprevisível, com potencial maior de ter linfonodos das regiões mediastinal superior e cervical envolvidos do que pacientes com adenocarcinoma que está sempre confinado ao esôfago inferior. Para os tumores do esôfago superior e médio, não foi informado a extensão da operação realizada nos trabalhos analisados, mas é improvável, nos países desenvolvidos e nos trabalhos multicêntricos, que dissecção linfonodal intratorácica agressiva tenha sido feita. A adição de radioterapia poderia ser benéfica neste aspecto. Os futuros trabalhos de terapêutica neo-adjuvante nos pacientes com câncer de esôfago necessitam estadiamento rigoroso (TC, PET, eco-endoscopia), além de controle de qualidade na operação com ao menos dissecção linfonodal em dois campos para que os efeitos da terapêutica adicional seja confiavelmente atribuída à neo-adjuvância e não a um tratamento cirúrgico inadequado.

\section{CONCLUSÃO}

O carcinoma epidermóide do esôfago apresenta prognóstico desfavorável apesar dos avanços no diagnóstico e terapêutica ocorrido nos últimos anos. Uma das estratégias na abordagem desta doença adotada por vários centros de tratamento foi o emprego terapêutico da quimioradioterapia seguida pela operação a fim de se melhorar os resultados na sobrevida dos pacientes e os resultados obtidos são promissores.

Tercioti V Jr, Lopes LR, Coelho Neto, JS, Andreollo NA - New aspects of the neo-adjuvant therapy in esophageal squamous cell carcinoma. A review of medical literature. ABCD Arq Bras Cir Dig 2009;22(1):33-40

ABSTRACT - Introduction - Esophageal neoplasm is the eighth more prevalent cancer in the general population, with high letality in spite of improvements in surgery in the last decades. The squamous cell carcinoma have higher prevalence in many countries and also in Brazil. Therefore, treatment strategies become target of study in many reference centres around the world. Aim - To identify the new aspects of the neo-adjuvant therapy in the esophageal squamous cell carcinoma. Methods - Bibliography review of scientific papers available at Medline and Cohchrane Database crossing the following headings: esophageal neoplasms, surgery, drug therapy, radiotherapy. Conclusion - Neo-adjuvant therapy is increasingly employed in patients with carcinoma of the esophagus in efforts to improve survival and quality of life of these patients.

HEADINGS - Esophageal neoplasms. Surgery. Drug therapy. Radiotherapy. 


\section{REFERÊNCIAS}

1. Ancona E, Ruol A, Castoro C, Chiarion-Sileni V, Merigliano S, Santi S et al. First-line chemotherapy improves the resection rate and long-term survival of locally advanced (T4, any N, M0) squamous cell carcinoma of the thoracic esophagus. Ann Surg 1997; 226(6):714-724.

2. Barros SGS, Ghisolfi ES, Luz LP, Barlem GG, Vidal RM, Wolff FH et al. Mate (chimarrão) é consumido em alta temperatura por população sob risco para o carcinoma epidermóide de esôfago. Arq Gastroenterol 2000;37(1):25-30.

3. Bosset JF, Gignoux M, Triboulet JP, Tiret E, Mantion G, Elias D et al. Chemoradiotherapy followed by surgery compared with surgery alone in squamous-cell cancer of the esophagus. N Engl J Med 1997; 337:161-7.

4. Burmeister BH, Smithers BM, Gebski V, Fitzgerald L, Simes RJ, Devitt P et al. Surgery alone versus chemoradiotherapy followed by surgery for resectable cancer of the oesophagus: a randomised controlled phase III trial. Lancet Oncol 2005; 6: 659-68.

5. Coia LR, Minsky BD, Berkey BA, John MJ, Haller D, Landry J et al. Outcome of patients receiving radiation for cancer of the esophagus: results of the 1992 1994 patterns of care study. J Clin Oncol 2000; 18(3): 455-462.

6. Estimativa 2008: Incidência de câncer no Brasil. Rio de Janeiro: INCA, 2007.

7. Fiorica F, Bona DD, Schepis F, Licata A, Shahied L, Venturi A et al. Preoperative chemoradiotherapy for oesophageal cancer: a systematic review and meta-analysis. Gut 2004; 53:925-930.

8. Gebski V, Burmeister B, Smithers BM, Foo K, Zalcberg J, Simes J. Survival benefits from neoadjuvant chemoradiotherapy or chemotherapy in oesophageal carcinoma: a meta-analysis. Lancet Oncol 2007; 8:226-234.

9. Greer SE, Goodney PP, Sutton JE, Birkmeyer JD. Neoadjuvant chemoradiotherapy for esophageal carcinoma: a meta-analysis. Surgery 2005; 137:172-7.

10. Hennequin C, Gayet B, Sauvanet A, Blazy A, Perniceni T, Pannis Y et al. Impact on survival of surgery after concomitant chemoradiotherapy for locally advanced cancers of the esophagus. Int J Radiat Oncol Biol Phys 2001; 49(3): 657-64.

11. Jemal A, Siegel R, Ward E, Murray T, Xu J, Thun MJ. Cancer Statistics, 2007. CA Cancer J Clin 2007; 57:43-66.

12. Jones DR, Detterbeck FC, Egan TM, Parker LA, Bernard SA, Tepper JE. Induction chemoradiotherapy followed by esophagectomy in patients with carcinoma of the esophagus. Ann Thorac Surg 1997; 64(1): 185-91.

13. Kleinberg L, Forastiere AA. Chemoradiation in the management of esophageal cancer. J Clin Oncol 2007;25:4110-4117.

14. Lagergren J, Bergström R, Lindgren A, Nyrén O. The role of tobacco, snuff and alcohol use in the aetiology of cancer of the oesophagus and gastric cardia. Int J Cancer 2000;85:340-346.

15. Le Prise E, Etienne PL, Meunier B, Maddern G, Hassel MB, Gedouin D et al. A randomized study of chemotherapy, radiation therapy, and surgery versus surgery for localized squamous cell carcinoma of the esophagus. Cancer 1994; 73(7): 1779-94.

16. Lee JL, Kim SB, Jung HJ, Park SI, Kim DK, Kim JH et al. Efficacy of neoadjuvant chemoradiotherapy in resectable esophageal in resectable esophageal squamous cell carcinoma. Acta Oncol 2003; 42(3): 207-11.
17. Makary MA, Kiernan PD, Sheridan MJ, Tonnesen G, Hetrick V, Vaughan B et al. Multimodality treatment for esophageal cancer: the role of surgery and neoadjuvant therapy. Am Surg 2003; 69(8):693-702.

18. Malthaner RA, Collin S, Fenlon D. Preoperative chemotherapy for resectable thoracic esophageal cancer. Cochrane Database of Systematic Reviews 2006, Issue 3. Art. No.:CD001556. DOI:10.1002/14651858.CD001556.pub2.

19. Malthaner RA, Wong RKS, Rumble RB, Zuraw L et al. Neoadjuvant or adjuvant therapy for resectable esophageal cancer: a systematic review and meta-analysis. BMC Med 2004;2:35.

20. Ministério da Saúde. IDB - 2005. Indicadores e Dados Básicos do Brasil. Homepage [www.datasus.gov.br].

21. Nabeya Y, Ochiai T, Matsubara H, Okazumi S, Shiratori T, Shuto K, Aoki T et al. Neoadjuvant chemoradiotherapy followed by esophagectomy for iniatially resectable squamous cell carcinoma of the esophagus with lymph node metástases. Dis Esophagus 2005; 18: 388-397.

22. Nakadi IE, Laethem JLV, Houben JJ, Gay F, Closset J, Houtte PV et al. Squamous cell carcinoma of the esophagus: multimodal therapy in locally advanced disease. World J Surg 2001; 26: 72-78.

23. Parkin DM, Bray F, Ferlay J, Pisani P. Global Cancer Statistics, 2002. CA Cancer J Clin 2005;55:74-108.

24. Pearson JG. The present status and future potential of radiotherapy in the management of esophageal cancer. Cancer 1977;39:882-890.

25. Queiroga RC, Pernambuco AP. Câncer de esôfago: epidemiologia, diagnóstico e tratamento. Rev Bras Cancerol 2006; 52(2):173-178.

26. Toita T, Ogawa K, Adachi G, Kakinohana Y, Nishikuramori Y, Iraha S et al. Concurrent chemoradiotherapy for squamous cell carcinoma of thoracic esophagus: feasibility and outcome of large regional field and high-dose external beam boost irradiation. Jpn J Clin Oncol 2001; 31(8):375-381.

27. Tytgat GNJ, Bartelink H, Bernards R, Giaccone G, Lanschot JJB, Offerhaus GJA et al. Cancer of the esophagus and gastric cardia: recent advances. Dis Esophagus 2004; 17:10-26.

28. Urba SG, Orringer MB, Turrisi A, Iannettoni M, Forastiere A, Strawderman $\mathrm{M}$. Randomized trial of preoperative chemoradiation versus surgery alone in patients with locoregional esophageal carcinoma. J Clin Oncol 2001; 19(2):305313.

29. Urschel JD, Vasan H. A meta-analysis of randomized controlled trials that compared neoadjuvant chemoradiation and surgery to surgery alone for resectable esophageal cancer. Am J Surg 2003; 185:538-543.

30. Zacherl J, Sendler A, Stein HJ, Ott K, Feith M, Jakesz R et al. Current status of neoadjuvant therapy for adenocarcinoma of the distal esophagus. World $\mathrm{J}$ Surg 2003; 27: 1067-1074.

31. Zhang X, Watson DI, Jamieson GG, Bessell JR, Devitt PG. Neoadjuvant chemoradiotherapy for esophageal carcinoma. Dis Esophagus 2005;18:104108.

Fonte de financiamento: não há Conflito de interesse: não há Recebido para publicação: 23/08/2008 Aceito para publicação: 22/12/2008 